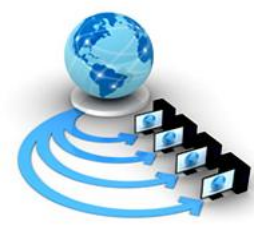

Volume 10, No. 6, November-December 2019

\title{
VECTOR OPERATION ON NODES OF PERFECT DIFERENCE NETWORK USING LOGICAL OPERATORS
}

\author{
Rakesh Kumar Katare ${ }^{1}$, Shrinivash Premikar ${ }^{1}$, Neha $\operatorname{Singh}^{1}$, Sunil Tiwar ${ }^{1}$, Charvi K ${ }^{2}$, \\ Department of Computer Science, Awadhesh Pratap Singh University, Rewa (MP) ${ }^{1}$ \\ Jawaharlal Nehru College of Technology, Ratahra, Rewa (MP) ${ }^{2}$
}

\begin{abstract}
In this paper we are exploring the bitwise connection between the nodes of a interconnection network. We are taking PDN as a model, First of all we are converting the interconnection network into its equivalent connectivity matrix .Then Row/Column vectors of connectivity matrix is used to present the value of a particular node of interconnection network which is shown in Figure 1 as state diagram of PDN which is $\delta=2$. Each bitwise vector shows connectivity with another node in position of bit 1 .The vectors also shows the mathematical property of PDN, it means the value of vector of a node in the connectivity matrix preserves the mathematical property of the topology. We assume that each node is connected to itself as a self loop in connectivity matrix. Therefore the diagonal matrix is always 1. The presence of 1's in a vector (Excluding the self loop) shows degree of the node. The connectivity and its complexity will be explored by using logical operators between the nodes of a PDN so that we can develop algorithms for automatic switching between two nodes automatically. In the due course of study we found many patterns of binary/logical relationship between the nodes which will be discussed in our future discussion in this paper.
\end{abstract}

Keywords: PDN, PDS, Interconnection Network, Connectivity Matrix.

\section{INTRODUCTION}

The Perfect difference set is discussed by J Singer in 1938 [11]. The formulation was in this terms of points and lines in a finite projective plane [1,2,3]. The Perfect Difference Set (PDS) considered for being develop into a interconnect network mainly through works of Parhami, Behrooz and Rakov, M.A [4,5]. In their, Perfect Difference Network (PDN) interconnection, they have shown that PDN interconnection scheme is best possible in the sense that it can cover the nodes with smallest node degree with network diameter 2. They have compared PDNs and some of their derivatives to interconnection networks with similar cost and performance with hypercube and its other variants[4]. Perfect difference networks are a robust high-performance interconnection network for parallel and distributed systems. A more exhaustive comparative study of perfect difference network and hypercube was done by Katare et al.,[6,10], based on topological structured properties. Topological properties of perfect difference network compared with the corresponding properties of hypercube by Katare et. al,[10]. In this technique, sparse linear system was implemented. It was proved that access function or routing function to map data on hypercube contains topological properties. The study of circuits based on the architecture of PDN is further taken forward by Katare et.al, july-25, 2013[12] in their research work on study of link utilization of PDN and Hypercube. They have shown that the circuits formed in PDN are a combination of odd and even length. Adjacency matrix of $\mathrm{n} x \mathrm{n}$ of PDN presented to study the link utilization and topological Properties[12]. In this paper we are converting PDN architecture in to equivalent Data structure for mapping into itself so that transition between nodes can be determined properly. The row vector which is equivalence to column vector can be used for logical operation for determining the binary relationship between nodes. The fabric nature of architecture can be properly defined for Development of algorithm to study the connectivity and Complexity of the architecture[5].

\subsection{Perfect Difference Set}

A set $\left\{\mathrm{s}_{0}, \mathrm{~s}_{1}, \ldots \ldots \mathrm{s}_{\delta}\right)$ of $\delta+1$ integers having the property that their $\delta^{2}+\delta$ differences, $0 \leq \mathrm{i} \neq \mathrm{j} \leq \delta$, are congruent modulo $\delta^{2}+\delta+1$, to the integers $1,2, \ldots \ldots, \delta^{2}+\delta$ in some order is a perfect difference set of order $\delta$. Perfect Difference Sets[11] are sometimes also called simple difference sets, given that they correspond to the special $\delta=$ 1 as a case of difference sets for which each of the possible differences is formed in exactly $\delta$ ways, where $\delta$ is a prime or power of prime and $\mathrm{n}=\delta^{2}+\delta+1$ and $\left(\mathrm{S}_{\mathrm{i}}-\mathrm{S}_{\mathrm{j}}\right)=\left(\delta^{2}+\delta\right) \bmod$ $\delta^{2}+\delta+1$.

\subsection{Perfect Difference Network}

The Perfect Difference set of each node of the PDN can be evaluated by the remainder theorem i.e.

$(\mathrm{N}=\mathrm{R}+\mathrm{D} * \mathrm{Q})$

Where $\mathrm{N}=$ Numerator, $\mathrm{R}=$ Remainder, $\mathrm{D}=$ Denominator and $\mathrm{Q}=\mathrm{Quotient}$

The above equation can be written as

Integer $=\left(\mathrm{S}_{\mathrm{i}}-\mathrm{S}_{\mathrm{j}}\right)+\left(\delta^{2}+\delta+1\right) * 1$

Where integer is a member of the set $\left(1,2, \ldots, \delta^{2}+\delta\right)$ and $\mathrm{S}_{\mathrm{i}^{-}}$ $\mathrm{S}_{\mathrm{j}}$ is numerator or the difference set.

So we can write as-

$\left(\mathrm{S}_{\mathrm{i}}-\mathrm{S}_{\mathrm{j}}\right)=$ (integer) $\bmod \delta^{2}+\delta+1[12]$

In the due case of study we are assuming that a node is connected to itself therefore the node is self connected in PDN. The following is the connectivity relation between nodes of a PDN.

$$
\begin{aligned}
& \quad \mathrm{i} \pm 1\left(0<\mathrm{i}<\delta^{2}+\delta\right) \\
& \quad \mathrm{i} \pm \mathrm{S}_{\mathrm{j}}(\bmod n) \text { for } 2 \leq \mathrm{j} \leq \delta
\end{aligned}
$$


This formulation is based on the definition of the numbered 0 to $\mathrm{n}$ - 1 , the direct mapping between nodes is represented by $i \pm 1$, which gives cordal ring pattern in network flow $\left(i \pm s_{j}(\bmod n)\right.$, for $\left.2 \leq j \leq \delta\right)$ means for each link from node $i$ to node $j,[3,6]$ the reverse link from node $j$ to node $\mathrm{i}$ is also exists, hence the network can be drawn as an undirected graph.

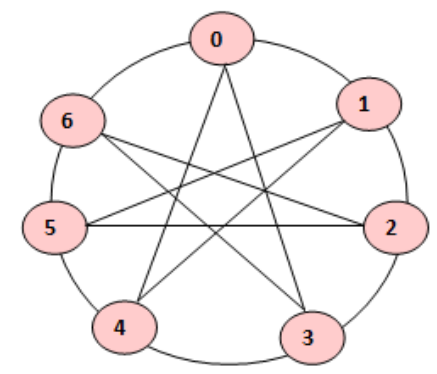

Fig.1: PDN having $\delta=2$ PDS $\left\{S_{0}, \mathrm{~S}_{1}, . ., \mathrm{S}_{\delta}\right\}$ There are $\mathrm{n}=\delta^{2}+\delta+1$ nodes,

Now the structural relation between nodes of a PDN can be connecting method in the following manner, where we are assuming that self loop for each node is considered for interconnection between processor \& Peripheral of one node.

\subsection{Connectivity Matrix}

In connectivity matrix if there is a connection between two nodes then its represented as 1 otherwise it is 0 . Symbolically connection matrix for PDN we have

$\mathrm{CM}_{\mathrm{ij}}=\left\{\begin{array}{l}\mathrm{i} \pm 1\left(0<\mathrm{i}<\delta^{2}+\delta\right)(\text { if node is } \\ \text { connected to itself or to another } \\ \text { node }) \\ 0\end{array}\right.$

$\leq \delta$ is a forward skip link of node $\mathrm{i}$ and a backward skip link of node $i+s_{j}(\bmod n)$.

since number of nodes for both columns and rows are equal. This defined the relation of nodes to itself.

Table 1 represents the connectivity matrix for PDN with $\delta=2$.

\begin{tabular}{|l|l|l|l|l|l|l|l|}
\hline & 0 & 1 & 2 & 3 & 4 & 5 & 6 \\
\hline 0 & 1 & 1 & 0 & 1 & 1 & 0 & 1 \\
\hline 1 & 1 & 1 & 1 & 0 & 1 & 1 & 0 \\
\hline 2 & 0 & 1 & 1 & 1 & 0 & 1 & 1 \\
\hline 3 & 1 & 0 & 1 & 1 & 1 & 0 & 1 \\
\hline 4 & 1 & 1 & 0 & 1 & 1 & 1 & 0 \\
\hline 5 & 0 & 1 & 1 & 0 & 1 & 1 & 1 \\
\hline 6 & 1 & 0 & 1 & 1 & 0 & 1 & 1 \\
\hline
\end{tabular}

Table 1: Connectivity matrix of nodes in a PDN having $\boldsymbol{\delta}=\mathbf{2}$

In this matrix Zero (0) represent that there is no information flow between the nodes and One (1) represent the information flow between the nodes. Such as node 0 can communicate with node 2 via node 1 or node 3 so in Table 1 $(0,2)$ contains 0. Similary node 0 can communicate directly with node 1 , so in the connectivity matrix in table $(0,1)$ contains 1 .

\subsection{The explanation of connectivity of as per vector of each node}

Perfect Difference Networks based on normal form of PDSs are special types of cordal rings. In the terminology of cordal rings, the links connecting consecutive nodes i- 1 and $i+1$ are ring links, while those that connect nonconsecutive nodes $\mathrm{i}$ and $\mathrm{i} \pm \mathrm{s}_{\mathrm{j}}(\bmod \mathrm{n})$, for $2 \leq \mathrm{j} \leq \delta$, are skip links or chords. The link connecting nodes $\mathrm{i}$ and $\mathrm{i}+\mathrm{s}_{\mathrm{j}}(\bmod \mathrm{n})$, for $2 \mathrm{j}$
- $\quad$ Node 0 is connected with node $(0,1,3,4,6)$

- $\quad$ Node 1 is connected with node $(0,1,2,4,5)$

- $\quad$ Node 2 is connected with node $(1,2,3,5,6)$

- $\quad$ Node 3 is connected with node $(1,2,3,4,6)$

- $\quad$ Node 4 is connected with node $(0,1,3,4,5)$

- $\quad$ Node 5 is connected with node $(1,2,4,5,6)$

- $\quad$ Node 6 is connected with node $(0,2,3,5,6)$

Table 1 Shown the connectivity of nodes of a PDN each row of the matrix where " 1 " shows the connectivity of nodes in " 0 " shows no connectivity between nodes is a vector used for logical operation for the investigation of the inter node connectivity of the network. Row and Column vectors are same which shows the symmetry of connectivity between processors. Here we are assuming \& considering the vector of a connectivity matrix as the value of a node, for example the vector of 0 node is (1101101) so this value is the value of node zero.

Now we are explaining the connectivity of each node as follows.

\begin{tabular}{|c|c|}
\hline Node Number & 0123456 \\
\hline 0 & $\{1,1,0,1,1,0,1\}$ \\
\hline 1 & $\{1,1,1,0,1,1,0\}$ \\
\hline 2 & $\{0,1,1,1,0,1,1\}$ \\
\hline 3 & $\{1,0,1,1,1,0,1\}$ \\
\hline 4 & $\{1,1,0,1,1,1,0\}$ \\
\hline 5 & $\{0,1,1,0,1,1,1\}$ \\
\hline 6 & $\{1,0,1,1,0,1,1\}$ \\
\hline
\end{tabular}

Table 2: Connectivity between processors in an interconnection network .

Now we are converting connectivity Matrix of PDN into its equivalent state diagram of PDN, interconnection network have been studied researcher for reduce the connectivity \& complexity of a set of nodes with particular architecture (PDN). Study of logical operation helps network flow to reaches from node $\mathrm{i}$ to any other 
nodes in minimum node connectivity. Here we present the some relation and show how the information flow may possible in the PDN when, one of node, connection fails. The logical AND operation between row vector of adjacent matrix of PDN gives the set of possible path for information network flow.

\begin{tabular}{|c|c|c|c|c|c|c|c|c|}
\hline \multirow{4}{*}{$\begin{array}{l}\text { the row ' } 0 \text { ' } \\
\text { the row ' } 3 \text { ' }\end{array}$} & \multirow{4}{*}{$\begin{array}{l}1101101 \\
1011101\end{array}$} & 1 & 1 & 0 & 1 & 1 & 0 & 1 \\
\hline & & 1 & 0 & 1 & 1 & 1 & 0 & 1 \\
\hline & & 1 & 0 & 0 & 1 & 1 & 0 & 1 \\
\hline & & 0 & $X$ & $x$ & 3 & 4 & $x$ & 6 \\
\hline
\end{tabular}

Here $(0,3)$ in express the direct connection \& $(0,4)$ $\&(0,6)$ in a alternate path for network flow. alternate path is useful when one of node connection in PDN architecture is failure, this relation also offer the benefits of full connectivity at a fault occurrence and a louver cost.

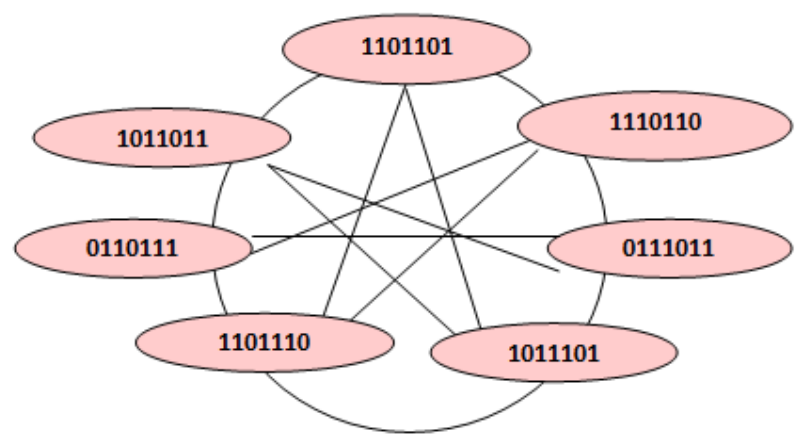

Fig. 2 state diagram of PDN with $\delta=2$.
Theorem: Vector of node 0 shows the connectivity between the nodes of PDN say (1101101) 0 Possible connectivity of this node is with $0,1,3,4,6$ if the connected node has value 1.

Proof:-The vector representation of each node shows the node connectivity as per perfect difference in Prefect Difference Network. For example vector of node 0 is (1101101) the presence of 1 shows the connectivity and 0 shows the disconnectivity.

$$
\left(\begin{array}{l}
0,1,2,3,4,5,6 \\
1,1,0,1,1,0,1
\end{array}\right)
$$

$(0,1,3,4,6)$ nodes are connected in PDS $\{0, \pm 1, \pm 3\}$.

The logical operation AND shows the connectivity of two nodes for example:

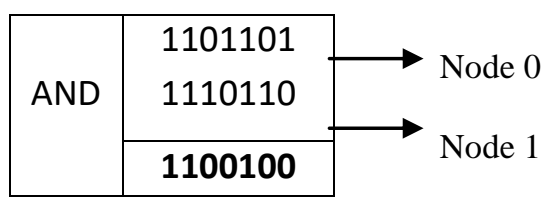

Node $(0,1,4)$ are connected to each other or in other words intersection of node $0 \&$ node 1 is $\{0,1,4\}$ both the operation are same as per the assumption of Discrete math's between Set Theory and mathematical logic.

Similarly other logical operations are OR, EX-OR, implication, Bi-Implication which can also be performed for finding the binary relation between the nodes of a PDN.

\begin{tabular}{|l|l|l|l|l|l|}
\hline Nodes & $\mathbf{0 1 2 3 4 5 6}$ & OR & EX-OR & Implication & Bi-Implication \\
\hline 0 & 1101101 & \multirow{2}{*}{1111111} & 0011011 & 1111111 & \multirow{2}{*}{1111111} \\
\hline 1 & 1110110 & & & \\
\hline
\end{tabular}

\section{Explanation of bit representation of Nodes}

In this section we are trying to establish the bitwise logical operation on the combination of vectors for find out the binary relation between the nodes, so that the binary relation can be proved for the study of connectivity and complexity of the flow of information in this architecture.

\subsection{Structural Pattern of architecture}

The operation "AND" gives the common nodes between two nodes where as OR gives the one of the nodes connectivity or both way connectivity. The equivalence gives both ways connectivity .on the other hand the implication gives validity of consequent. The Ex-OR gives the two way switching.

The following tables show the binary logical relation between the nodes.

\begin{tabular}{|c|c|c|c|c|c|c|c|c|c|}
\hline Node & AND & $\begin{array}{c}\text { Possible } \\
\text { Connectivity }\end{array}$ & $\begin{array}{c}\text { Patterns of } \\
\text { PDN } \\
\text { connectivity }\end{array}$ & OR & $\begin{array}{c}\text { Possible } \\
\text { Connectivity }\end{array}$ & $\begin{array}{c}\text { patterns of } \\
\text { PDN } \\
\text { connectivity }\end{array}$ & EX-OR & $\begin{array}{c}\text { Possible } \\
\text { Connectivity }\end{array}$ & $\begin{array}{l}\text { Patterns of } \\
\text { PDN } \\
\text { connectivity }\end{array}$ \\
\hline 0 & 1101101 & \multirow[t]{3}{*}{$0,1,3,4,6$} & \multirow[t]{3}{*}{5} & 1101101 & \multirow[t]{3}{*}{$0,1,3,4,6$} & \multirow[t]{3}{*}{5} & 1101101 & \multirow{3}{*}{$\begin{array}{l}\text { no nodes } \\
\text { are } \\
\text { connected }\end{array}$} & \multirow[t]{3}{*}{0} \\
\hline \multirow[t]{2}{*}{0} & 1101101 & & & 1101101 & & & 1101101 & & \\
\hline & 1101101 & & & 1101101 & & & 0000000 & & \\
\hline 0 & 1101101 & \multirow[t]{3}{*}{$0,1,4$} & \multirow[t]{3}{*}{3} & 1101101 & \multirow{3}{*}{$\begin{array}{l}\text { all nodes } \\
\text { are } \\
\text { connected }\end{array}$} & \multirow[t]{3}{*}{7} & 1101101 & \multirow[t]{3}{*}{$2,3,5,6$} & \multirow[t]{3}{*}{4} \\
\hline 1 & 1110110 & & & 1110110 & & & 1110110 & & \\
\hline & 1100100 & & & 1111111 & & & 0011011 & & \\
\hline
\end{tabular}


Rakesh Kumar Katare et al, International Journal of Advanced Research in Computer Science, 10 (6), Nov-Dec 2019,29-39

\begin{tabular}{|c|c|c|c|c|c|c|c|c|c|}
\hline 0 & 1101101 & \multirow[t]{3}{*}{$1,3,6$} & \multirow[t]{3}{*}{3} & 1101101 & \multirow{3}{*}{$\begin{array}{l}\text { all nodes } \\
\text { are } \\
\text { connected }\end{array}$} & \multirow[t]{3}{*}{7} & 1101101 & \multirow[t]{3}{*}{$0,2,4,5$} & \multirow[t]{3}{*}{4} \\
\hline \multirow[t]{2}{*}{2} & 0111011 & & & 0111011 & & & 0111011 & & \\
\hline & 1001101 & & & 1111111 & & & 1010110 & & \\
\hline 0 & 1101101 & \multirow[t]{3}{*}{$0,3,4,6$} & \multirow[t]{3}{*}{4} & 1101101 & \multirow[t]{3}{*}{$0,1,2,3,4,6$} & \multirow[t]{3}{*}{6} & 1101101 & \multirow[t]{3}{*}{1,2} & \multirow[t]{3}{*}{2} \\
\hline \multirow[t]{2}{*}{3} & 1011101 & & & 1011101 & & & 1011101 & & \\
\hline & 1001101 & & & 1111101 & & & 0110000 & & \\
\hline 0 & 1101101 & \multirow[t]{3}{*}{$0,1,3,4$} & \multirow[t]{3}{*}{4} & 1101101 & \multirow[t]{3}{*}{$0,1,3,4,5,6$} & \multirow[t]{3}{*}{6} & 1101101 & \multirow[t]{3}{*}{5,6} & \multirow[t]{3}{*}{2} \\
\hline \multirow[t]{2}{*}{4} & 1101110 & & & 1101110 & & & 1101110 & & \\
\hline & 1101100 & & & 1101111 & & & 0000011 & & \\
\hline 0 & 1101101 & \multirow[t]{3}{*}{$1,4,6$} & 3 & 1101101 & all nodes & 7 & 1101101 & $0,2,3,5$ & 4 \\
\hline 5 & 0110111 & & & 0110111 & $\begin{array}{c}\text { are } \\
\text { connected }\end{array}$ & & 0110111 & & \\
\hline & 100101 & & & 1111111 & & & 1011010 & & \\
\hline 0 & 1101101 & $0,3,6$ & 3 & 1101101 & all nodes & 7 & 1101101 & $1,2,4,5$ & 4 \\
\hline 6 & 1011011 & & & 1011011 & $\begin{array}{c}\text { are } \\
\text { connected }\end{array}$ & & 1011011 & & \\
\hline & 1001001 & & & 1111111 & & & 0110110 & & \\
\hline 1 & 1110110 & $0,1,2,4,5$ & 5 & 1110110 & $0,1,2,4,5$ & 5 & 1110110 & no nodes & 0 \\
\hline 1 & 1110110 & & & 1110110 & & & 1110110 & $\begin{array}{c}\text { are } \\
\text { connected }\end{array}$ & \\
\hline & 1110110 & & & 1110110 & & & 0000000 & & \\
\hline 1 & 1110110 & $1,2,5$ & 3 & 1110110 & all nodes & 7 & 1110110 & $0,3,4,6$ & 4 \\
\hline 2 & 0111011 & & & 0111011 & & & 0111011 & & \\
\hline & 0110010 & & & 1111111 & & & 1001101 & & \\
\hline 1 & 1110110 & $0,2,4$ & 3 & 1110110 & all nodes & 7 & 1110110 & $1,3,5,6$ & 4 \\
\hline 3 & 1011101 & & & 1011101 & & & 1011101 & & \\
\hline & 1010100 & & & 1111111 & & & 0101011 & & \\
\hline 1 & 1110110 & $0,1,4,5$ & 4 & 1110110 & $0,1,2,3,4,5$ & 6 & 1110110 & 2,3 & 2 \\
\hline 4 & 1101110 & & & 1101110 & & & 1101110 & & \\
\hline & 1100110 & & & 1111110 & & & 0011000 & & \\
\hline 1 & 1110110 & $1,2,4,5$ & 4 & 1110110 & $0,1,2,4,5,6$ & 6 & 1110110 & 0,6 & 2 \\
\hline 5 & 0110111 & & & 0110111 & & & 0110111 & & \\
\hline & 0110110 & & & 1110111 & & & 1000001 & & \\
\hline 1 & 1110110 & $0,2,5$ & 3 & 1110110 & all nodes & 7 & 1110110 & $1,3,4,6$ & 4 \\
\hline 6 & 1011011 & & & 1011011 & are & & 1011011 & & \\
\hline & 1010010 & & & 1111111 & connected & & 0101101 & & \\
\hline 2 & 0111011 & $1,2,3,5,6$ & 5 & 0111011 & $1,2,3,5,6$ & 5 & 0111011 & $\begin{array}{c}\text { no nodes } \\
\text { are }\end{array}$ & 0 \\
\hline 2 & 0111011 & & & 0111011 & & & 0111011 & connected & \\
\hline & 0111011 & & & 0111011 & & & 0000000 & & \\
\hline 2 & 0111011 & $2,3,6$ & 3 & 0111011 & all nodes & 7 & 0111011 & $0,1,4,5$ & 4 \\
\hline 3 & 1011101 & & & 1011101 & & & 1011101 & & \\
\hline & 0011001 & & & 1111111 & & & 1100110 & & \\
\hline 2 & 0111011 & $1,3,5$ & 3 & 0111011 & all nodes & 7 & 0111011 & $0,2,4,6$ & 4 \\
\hline 4 & 1101110 & & & 1101110 & & & 1101110 & & \\
\hline & 0101010 & & & 1111111 & & & 1010101 & & \\
\hline 2 & 0111011 & $1,2,5,6$ & 4 & 0111011 & $1,2,3,4,5,6$ & 6 & 0111011 & 3,4 & 2 \\
\hline 5 & 0110111 & & & 0110111 & & & 0110111 & & \\
\hline
\end{tabular}


Rakesh Kumar Katare et al, International Journal of Advanced Research in Computer Science, 10 (6), Nov-Dec 2019,29-39

\begin{tabular}{|c|c|c|c|c|c|c|c|c|c|}
\hline & 0110011 & & & 0111111 & & & 0001100 & & \\
\hline 2 & 0111011 & \multirow[t]{3}{*}{$2,3,5,6$} & \multirow[t]{3}{*}{4} & 0111011 & \multirow[t]{3}{*}{$0,1,2,3,5,6$} & \multirow[t]{3}{*}{6} & 0111011 & \multirow[t]{3}{*}{0,1} & \multirow[t]{3}{*}{2} \\
\hline \multirow[t]{2}{*}{6} & 1011011 & & & 1011011 & & & 1011011 & & \\
\hline & 0011011 & & & 1111011 & & & 1100000 & & \\
\hline 3 & 1011101 & \multirow[t]{3}{*}{$0,2,3,4,6$} & \multirow[t]{3}{*}{5} & 1011101 & \multirow[t]{3}{*}{$0,2,3,4,6$} & \multirow[t]{3}{*}{5} & 1011101 & \multirow{3}{*}{$\begin{array}{l}\text { no nodes } \\
\text { are } \\
\text { connected }\end{array}$} & \multirow[t]{3}{*}{0} \\
\hline \multirow[t]{2}{*}{3} & 1011101 & & & 1011101 & & & 1011101 & & \\
\hline & 1011101 & & & 1011101 & & & 0000000 & & \\
\hline 3 & 1011101 & \multirow[t]{3}{*}{$0,3,4$} & \multirow[t]{3}{*}{3} & 1011101 & \multirow{3}{*}{$\begin{array}{l}\text { all nodes } \\
\text { are } \\
\text { connected }\end{array}$} & \multirow[t]{3}{*}{7} & 1011101 & \multirow[t]{3}{*}{$1,2,5,6$} & \multirow[t]{3}{*}{4} \\
\hline \multirow[t]{2}{*}{4} & 1101110 & & & 1101110 & & & 1101110 & & \\
\hline & 1001100 & & & 1111111 & & & 0110011 & & \\
\hline 3 & 1011101 & \multirow[t]{3}{*}{$2,4,6$} & \multirow[t]{3}{*}{3} & 1011101 & \multirow{3}{*}{$\begin{array}{l}\text { all nodes } \\
\text { are } \\
\text { connected }\end{array}$} & \multirow[t]{3}{*}{7} & 1011101 & \multirow[t]{3}{*}{$0,1,4,5$} & \multirow[t]{3}{*}{4} \\
\hline 5 & 0110111 & & & 0110111 & & & 0110111 & & \\
\hline & 0010101 & & & 1111111 & & & 1101010 & & \\
\hline 3 & 1011101 & $0,2,3,6$ & 4 & 1011101 & $0,2,3,4,5,6$ & 6 & 1011101 & 4,5 & 2 \\
\hline 6 & 1011011 & & & 1011011 & & & 1011011 & & \\
\hline & 1011001 & & & 1011111 & & & 0000110 & & \\
\hline 4 & 1101110 & $0,1,3,4,5$ & 5 & 1101110 & $0,1,3,4,5$ & 5 & 1101110 & no nodes & 0 \\
\hline 4 & 1101110 & & & 1101110 & & & 1101110 & are & \\
\hline & 1101110 & & & 1101110 & & & 0000000 & & \\
\hline 4 & 1101110 & $1,4,5$ & 3 & 1101110 & all nodes & 7 & 1101110 & $0,2,3,6$ & 4 \\
\hline 5 & 0110111 & & & 0110111 & & & 0110111 & & \\
\hline & 0100110 & & & 1111111 & & & 1011001 & & \\
\hline 4 & 1101110 & $0,3,5$ & 3 & 1101110 & all nodes & 7 & 1101110 & $1,2,4,6$ & 4 \\
\hline 6 & 1011011 & & & 1011011 & & & 1011011 & & \\
\hline & 1001010 & & & 1111111 & & & 0110101 & & \\
\hline 5 & 0110111 & $1,2,4,5,6$ & 5 & 0110111 & $1,2,4,5,6$ & 5 & 0110111 & no nodes & 0 \\
\hline 5 & 0110111 & & & 0110111 & & & 0110111 & & \\
\hline & 0110111 & & & 0110111 & & & 0000000 & & \\
\hline 5 & 0110111 & $2,5,6$ & 3 & 0110111 & all nodes & 7 & 0110111 & $0,1,3,4$ & 4 \\
\hline 6 & 1011011 & & & 1011011 & & & 1011011 & & \\
\hline & 0010011 & & & 1111111 & & & 1101100 & & \\
\hline 6 & 1011011 & $0,2,3,5,6$ & 5 & 1011011 & $0,2,3,5,6$ & 5 & 1011011 & no nodes & 0 \\
\hline 6 & 1011011 & & & 1011011 & & & 1011011 & $\begin{array}{c}\text { are } \\
\text { connected }\end{array}$ & \\
\hline & 1011011 & & & 1011011 & & & 0000000 & connected & \\
\hline
\end{tabular}

Table-3 logical operation with AND, OR, EX-OR

\begin{tabular}{|c|c|c|c|c|c|c|}
\hline Node & Implication & $\begin{array}{l}\text { Possible } \\
\text { Connectivity }\end{array}$ & $\begin{array}{l}\text { Patterns of PDN } \\
\text { connectivity }\end{array}$ & $\begin{array}{c}\text { Bi- } \\
\text { Implication }\end{array}$ & $\begin{array}{l}\text { Possible } \\
\text { Connectivity }\end{array}$ & $\begin{array}{l}\text { Patterns of PDN } \\
\text { connectivity }\end{array}$ \\
\hline 0 & 1101101 & \multirow{3}{*}{$\begin{array}{c}\text { all nodes are } \\
\text { connected }\end{array}$} & \multirow[t]{3}{*}{ ( } & 1101101 & \multirow{3}{*}{$\begin{array}{c}\text { all nodes are } \\
\text { connected }\end{array}$} & \multirow[t]{3}{*}{ 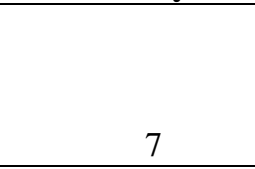 } \\
\hline \multirow[t]{2}{*}{0} & 1101101 & & & 1101101 & & \\
\hline & 1111111 & & & 1111111 & & \\
\hline 0 & 1101101 & \multirow[b]{3}{*}{$0,1,2,4,5$} & \multirow[b]{3}{*}{5} & 1101101 & \multirow[b]{3}{*}{$0,1,4$} & \multirow[b]{3}{*}{3} \\
\hline 1 & 1110110 & & & 1110110 & & \\
\hline & 1110110 & & & 1100100 & & \\
\hline 0 & 1101101 & \multirow[b]{2}{*}{$1,2,3,5,6$} & \multirow[b]{2}{*}{5} & 1101101 & \multirow[b]{2}{*}{$1,3,6$} & \multirow[b]{2}{*}{3} \\
\hline 2 & 0111011 & & & 0111011 & & \\
\hline
\end{tabular}


Rakesh Kumar Katare et al, International Journal of Advanced Research in Computer Science, 10 (6), Nov-Dec 2019,29-39

\begin{tabular}{|c|c|c|c|c|c|c|}
\hline & 0111011 & & & 0101001 & & \multirow[b]{4}{*}{5} \\
\hline 0 & 1101101 & \multirow[b]{3}{*}{$0,2,3,4,5,6$} & \multirow[b]{3}{*}{6} & 1101101 & \multirow[b]{3}{*}{$0,3,4,5,6$} & \\
\hline \multirow[t]{2}{*}{3} & 1011101 & & & 1011101 & & \\
\hline & 1011111 & & & 1001111 & & \\
\hline 0 & 1101101 & \multirow[b]{3}{*}{$0,1,2,3,4,5$} & \multirow[b]{3}{*}{6} & 1101101 & \multirow[b]{3}{*}{$0,1,2,3,4$} & \multirow[b]{3}{*}{5} \\
\hline \multirow[t]{2}{*}{4} & 1101110 & & & 1101110 & & \\
\hline & 1111110 & & & 1111100 & & \\
\hline 0 & 1101101 & \multirow[b]{3}{*}{$1,2,4,5,6$} & \multirow[b]{3}{*}{5} & 1101101 & \multirow[b]{3}{*}{$1,4,6$} & \multirow[b]{3}{*}{3} \\
\hline \multirow[t]{2}{*}{5} & 0110111 & & & 0110111 & & \\
\hline & 0110111 & & & 0100101 & & \\
\hline 0 & 1101101 & \multirow[b]{3}{*}{$0,2,3,5,6$} & \multirow[b]{3}{*}{5} & 1101101 & \multirow[b]{3}{*}{$0,3,6$} & \multirow[b]{3}{*}{3} \\
\hline 6 & 1011011 & & & 1011011 & & \\
\hline & 1011011 & & & 1001001 & & \\
\hline 1 & 1110110 & \multirow{3}{*}{$\begin{array}{c}\text { all nodes are } \\
\text { connected }\end{array}$} & \multirow[b]{3}{*}{7} & 1110110 & \multirow{3}{*}{$\begin{array}{l}\text { all nodes are } \\
\text { connected }\end{array}$} & \multirow[b]{3}{*}{7} \\
\hline 1 & 1110110 & & & 1110110 & & \\
\hline & 1111111 & & & 1111111 & & \\
\hline 1 & 1110110 & & & 1110110 & & \\
\hline 2 & 0111011 & & & 0111011 & & \\
\hline & 0111011 & $1,2,3,5,6$ & 5 & 0110010 & $1,2,5$ & 3 \\
\hline 1 & 1110110 & & & 1110110 & & 3 \\
\hline 3 & 1011101 & & & 1011101 & & 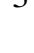 \\
\hline & 1011101 & $0,2,3,4,6$ & 5 & 1010100 & $0,2,4$ & \\
\hline 1 & 1110110 & & & 1110110 & & \\
\hline 4 & 1101110 & & & 1101110 & & \\
\hline & 1101111 & $0,1,3,4,5,6$ & 6 & 1100111 & ] $0,1,4,5,6$ & 5 \\
\hline 1 & 1110110 & & & 1110110 & & \\
\hline 5 & 0110111 & & & 0110111 & & \\
\hline & 0111111 & $1,2,3,4,5,6$ & 6 & 0111110 & $1,2,3,4,5$ & 5 \\
\hline 1 & 1110110 & & & 1110110 & & \\
\hline 6 & 1011011 & & & 1011011 & & \\
\hline & 1011011 & $0,2,3,5,6$ & 5 & 1010010 & $0,2,5$ & 3 \\
\hline 2 & 0111011 & & 7 & 0111011 & & \\
\hline 2 & 0111011 & all nodes are & 1 & 0111011 & all nodes are & \\
\hline & 1111111 & connected & & 1111111 & connected & 7 \\
\hline 2 & 0111011 & & & 0111011 & & \\
\hline 3 & 1011101 & & & 1011101 & & \\
\hline & 1011101 & $0,2,3,4,6$ & 5 & 0011001 & $2,3,6$ & 3 \\
\hline 2 & 0111011 & & & 0111011 & & \\
\hline 4 & 1101110 & & & 1101110 & & \\
\hline & 1101110 & $0,1,3,4,5$ & 5 & 0101010 & $1,3,5$ & 3 \\
\hline 2 & 0111011 & & & 0111011 & & \\
\hline 5 & 0110111 & & & 0110111 & & \\
\hline & 1110111 & $0,1,2,4,5,6$ & 6 & 1110011 & $0,1,2,5,6$ & 5 \\
\hline 2 & 0111011 & & & 0111011 & & \\
\hline 6 & 1011011 & & & 1011011 & & \\
\hline & 1011111 & $0,2,3,4,5,6$ & 6 & 0011111 & $2,3,4,5,6$ & 5 \\
\hline
\end{tabular}




\begin{tabular}{|c|c|c|c|c|c|c|}
\hline 3 & 1011101 & \multirow{3}{*}{$\begin{array}{l}\text { all nodes are } \\
\text { connected }\end{array}$} & \multirow[b]{3}{*}{7} & 1011101 & \multirow{3}{*}{$\begin{array}{l}\text { all nodes are } \\
\text { connected }\end{array}$} & \multirow[b]{3}{*}{7} \\
\hline \multirow[t]{2}{*}{3} & 1011101 & & & 1011101 & & \\
\hline & 1111111 & & & 1111111 & & \\
\hline 3 & 1011101 & \multirow[b]{3}{*}{$0,1,3,4,5$} & \multirow[b]{3}{*}{5} & 1011101 & \multirow[b]{3}{*}{$0,3,4$} & \multirow[b]{3}{*}{3} \\
\hline \multirow[t]{2}{*}{4} & 1101110 & & & 1101110 & & \\
\hline & 1101110 & & & 1001100 & & \\
\hline 3 & 1011101 & \multirow[b]{3}{*}{$0,2,4,5,6$} & \multirow[b]{3}{*}{5} & 1011101 & \multirow[b]{3}{*}{$2,4,6$} & \multirow[b]{3}{*}{3} \\
\hline \multirow[t]{2}{*}{5} & 0110111 & & & 0110111 & & \\
\hline & 1010111 & & & 0010101 & & \\
\hline 3 & 1011101 & \multirow[b]{3}{*}{$0,1,2,3,5,6$} & \multirow[b]{3}{*}{6} & 1011101 & \multirow[b]{3}{*}{$0,1,2,3,6$} & \multirow[b]{3}{*}{5} \\
\hline \multirow[t]{2}{*}{6} & 1011011 & & & 1011011 & & \\
\hline & 1111011 & & & 1111001 & & \\
\hline 4 & 1101110 & \multirow{3}{*}{$\begin{array}{l}\text { all nodes are } \\
\text { connected }\end{array}$} & \multirow[b]{3}{*}{7} & 1101110 & \multirow{3}{*}{$\begin{array}{l}\text { all nodes are } \\
\text { connected }\end{array}$} & \multirow[b]{3}{*}{7} \\
\hline \multirow[t]{2}{*}{4} & 1101110 & & & 1101110 & & \\
\hline & 1111111 & & & 1111111 & & \\
\hline 4 & 1101110 & \multirow[b]{3}{*}{$1,2,4,5,6$} & \multirow[b]{3}{*}{5} & 1101110 & \multirow[b]{3}{*}{$1,4,5$} & \multirow[b]{3}{*}{3} \\
\hline \multirow[t]{2}{*}{5} & 0110111 & & & 0110111 & & \\
\hline & 0110111 & & & 0100110 & & \\
\hline 4 & 1101110 & & & 1101110 & \multirow[b]{3}{*}{$0,3,5$} & \\
\hline 6 & 1011011 & & & 1011011 & & 3 \\
\hline & 1011011 & $0,2,3,5,6$ & 5 & 1001010 & & \\
\hline 5 & 0110111 & & & 0110111 & & \\
\hline 5 & $\begin{array}{l}0110111 \\
1111111\end{array}$ & $\begin{array}{l}\text { all nodes are } \\
\text { connected }\end{array}$ & 7 & $\begin{array}{l}0110111 \\
1111111\end{array}$ & $\begin{array}{l}\text { all nodes are } \\
\text { connected }\end{array}$ & 7 \\
\hline 5 & 0110111 & & & 0110111 & & \\
\hline 6 & 1011011 & & & 1011011 & & \\
\hline & 1011101 & $0,2,3,4,6$ & 5 & 0010101 & $2,4,6$ & 3 \\
\hline 6 & 1011011 & & & 1011011 & & \\
\hline 6 & 1011011 & all nodes are & & 1011011 & all nodes are & \\
\hline & 1111111 & connected & 7 & 1111111 & connected & 7 \\
\hline
\end{tabular}

Table-3(Continue) logical operation with Implication and Bi-Implication

2.2 Finding Connectivity links, Missing link between nodes of $\operatorname{PDN}$ as per $\operatorname{PDS}=\{-3,-1,0,1,3\}$

\begin{tabular}{|c|c|c|c|c|c|c|c|c|c|c|c|c|}
\hline A & $\mathbf{B}$ & connected nodes & $\mathbf{A + 0}$ & $\mathbf{A + 1}$ & $\mathbf{A - 1}$ & $\mathbf{A + 3}$ & $\mathbf{A - 3}$ & $\mathbf{B + 0}$ & $\mathbf{B + 1}$ & $\mathbf{B}-\mathbf{1}$ & $\mathbf{B}+\mathbf{3}$ & $\mathbf{B}-\mathbf{3}$ \\
\hline $\mathbf{0}$ & $\mathbf{0}$ & $\mathbf{0 , 1 , 3 , 4 , 6}$ & 0 & 1 & 6 & 3 & 4 & 0 & 1 & 6 & 4 & 0 \\
\hline $\mathbf{0}$ & $\mathbf{1}$ & $\mathbf{0 , 1 , 4}$ & 0 & 1 & $*$ & $*$ & 4 & 1 & $*$ & 0 & 4 & $*$ \\
\hline $\mathbf{0}$ & $\mathbf{2}$ & $\mathbf{1 , 3 , 6}$ & 0 & 1 & 6 & 3 & $*$ & $*$ & 3 & 1 & $*$ & 6 \\
\hline $\mathbf{0}$ & $\mathbf{3}$ & $\mathbf{0 , 3 , 4 , 6}$ & 0 & 6 & $*$ & 4 & 3 & 3 & $*$ & 4 & 6 & 0 \\
\hline $\mathbf{0}$ & $\mathbf{4}$ & $\mathbf{0 , 1 , 3 , 4}$ & 0 & 1 & $*$ & 3 & 4 & 4 & $*$ & 4 & 0 & 1 \\
\hline $\mathbf{0}$ & $\mathbf{5}$ & $\mathbf{1 , 4 , 6}$ & $*$ & 1 & 6 & $*$ & 4 & $*$ & 6 & 4 & 1 & $*$ \\
\hline $\mathbf{0}$ & $\mathbf{6}$ & $\mathbf{0 , 3 , 6}$ & 0 & & 6 & 3 & $*$ & 6 & 0 & $*$ & $*$ & 3 \\
\hline $\mathbf{1}$ & $\mathbf{1}$ & $\mathbf{0 , 1 , 2 , 4 , 5}$ & 1 & 2 & 0 & 4 & 5 & 1 & 2 & 0 & 4 & 5 \\
\hline $\mathbf{1}$ & $\mathbf{2}$ & $\mathbf{1 , 2 , 5}$ & 1 & 2 & $*$ & $*$ & 5 & 2 & $*$ & 1 & 5 & $*$ \\
\hline $\mathbf{1}$ & $\mathbf{3}$ & $\mathbf{0 , 2 , 4}$ & $*$ & 2 & 0 & 4 & $*$ & $*$ & 4 & 2 & $*$ & 0 \\
\hline $\mathbf{1}$ & $\mathbf{4}$ & $\mathbf{0 , 1 , 4 , 5}$ & 1 & $*$ & 0 & 4 & 5 & 4 & 5 & $*$ & $*$ & 1 \\
\hline $\mathbf{1}$ & $\mathbf{5}$ & $\mathbf{1 , 2 , 4 , 5}$ & 1 & 2 & $*$ & 4 & 5 & 5 & $*$ & 4 & 1 & 2 \\
\hline
\end{tabular}




\begin{tabular}{|l|l|c|c|c|c|c|c|c|c|c|c|c|}
$\mathbf{1}$ & $\mathbf{6}$ & $\mathbf{0 , 2 , 5}$ & 1 & 2 & 0 & $*$ & 5 & $*$ & 0 & 5 & 2 & $*$ \\
\hline $\mathbf{2}$ & $\mathbf{2}$ & $\mathbf{1 , 2 , 3 , 5 , 6}$ & 2 & 3 & 1 & 5 & 6 & 2 & 3 & 1 & 5 & 6 \\
\hline $\mathbf{2}$ & $\mathbf{3}$ & $\mathbf{2 , 3 , 6}$ & 2 & 3 & $*$ & $*$ & 6 & 3 & & 2 & 6 & $*$ \\
\hline $\mathbf{2}$ & $\mathbf{4}$ & $\mathbf{1 , 3 , 5}$ & 2 & 3 & 1 & 5 & $*$ & $*$ & 5 & 3 & $*$ & 1 \\
\hline $\mathbf{2}$ & $\mathbf{5}$ & $\mathbf{1 , 2 , 5 , 6}$ & 2 & $*$ & 1 & 5 & 6 & 5 & 6 & $*$ & 1 & 2 \\
\hline $\mathbf{2}$ & $\mathbf{6}$ & $\mathbf{2 , 3 , 5 , 6}$ & 2 & 3 & $*$ & 5 & 6 & 6 & $*$ & 5 & 2 & 3 \\
\hline $\mathbf{3}$ & $\mathbf{3}$ & $\mathbf{0 , 2 , 3 , 4 , 6}$ & 3 & 4 & 2 & 6 & 0 & 3 & 4 & 2 & 6 & 0 \\
\hline $\mathbf{3}$ & $\mathbf{4}$ & $\mathbf{0 , 3 , 4}$ & 3 & 4 & $*$ & $*$ & 0 & 4 & $*$ & 3 & 0 & $*$ \\
\hline $\mathbf{3}$ & $\mathbf{5}$ & $\mathbf{2 , 4 , 6}$ & $*$ & 4 & 2 & 6 & $*$ & $*$ & 6 & 4 & $*$ & 2 \\
\hline $\mathbf{3}$ & $\mathbf{6}$ & $\mathbf{0 , 2 , 3 , 6}$ & 3 & $*$ & 2 & 6 & 0 & 6 & 0 & & 2 & 3 \\
\hline $\mathbf{4}$ & $\mathbf{4}$ & $\mathbf{0 , 1 , 3 , 4 , 5}$ & 4 & 5 & 3 & 0 & 1 & 4 & 5 & 3 & 0 & 1 \\
\hline $\mathbf{4}$ & $\mathbf{5}$ & $\mathbf{1 , 4 , 5}$ & 4 & 5 & $*$ & $*$ & 1 & 5 & $*$ & 4 & 1 & $*$ \\
\hline $\mathbf{4}$ & $\mathbf{6}$ & $\mathbf{0 , 3 , 5}$ & $*$ & 5 & 3 & 0 & 1 & $*$ & 0 & 5 & $*$ & 3 \\
\hline $\mathbf{5}$ & $\mathbf{5}$ & $\mathbf{1 , 2 , 4 , 5 , 6}$ & 5 & 6 & 4 & 1 & 2 & 5 & 6 & 4 & 1 & 2 \\
\hline $\mathbf{5}$ & $\mathbf{6}$ & $\mathbf{2 , 5 , 6}$ & 5 & $*$ & 6 & 2 & $*$ & 6 & 5 & $*$ & 2 & $*$ \\
\hline $\mathbf{6}$ & $\mathbf{6}$ & $\mathbf{0 , 2 , 3 , 5 , 6}$ & 6 & 5 & 0 & 3 & 2 & 6 & 5 & 0 & 3 & 2 \\
\hline
\end{tabular}

\section{Based on PDS $(0, \pm 1, \pm 3)$ For OR logical operation}

\begin{tabular}{|c|c|c|c|c|c|c|c|c|c|c|c|c|}
\hline $\mathbf{A}$ & $\mathbf{B}$ & $\mathbf{c o n n e c t e d ~ n o d e s ~}$ & $\mathbf{A + 0}$ & $\mathbf{A + 1}$ & $\mathbf{A - 1}$ & $\mathbf{A + 3}$ & $\mathbf{A - 3}$ & $\mathbf{B + 0}$ & $\mathbf{B + 1}$ & $\mathbf{B}-\mathbf{1}$ & $\mathbf{B}+\mathbf{3}$ & $\mathbf{B}-\mathbf{3}$ \\
\hline $\mathbf{0}$ & $\mathbf{0}$ & $\mathbf{0 , 1 , 3 , 4 , 6}$ & 0 & 1 & 6 & 3 & 4 & 0 & 1 & 6 & 4 & 0 \\
\hline $\mathbf{0}$ & $\mathbf{1}$ & $\mathbf{0 , 1 , 2 , 3 , 4 , 5 , 6}$ & 0 & 1 & 6 & 3 & 4 & 1 & 2 & 0 & 4 & 5 \\
\hline $\mathbf{0}$ & $\mathbf{2}$ & $\mathbf{0 , 1 , 2 , 3 , 4 , 5 , 6}$ & 0 & 1 & 6 & 3 & 4 & 2 & 3 & 1 & 5 & 6 \\
\hline $\mathbf{0}$ & $\mathbf{3}$ & $\mathbf{0 , 1 , 2 , 3 , 4 , 6}$ & 0 & 6 & 1 & 4 & 3 & 3 & 2 & 4 & 6 & 0 \\
\hline $\mathbf{0}$ & $\mathbf{4}$ & $\mathbf{0 , 1 , 3 , 4 , 5 , 6}$ & 0 & 1 & 6 & 3 & 4 & 4 & 5 & 3 & 0 & 1 \\
\hline $\mathbf{0}$ & $\mathbf{5}$ & $\mathbf{0 , 1 , 2 , 3 , 4 , 5 , 6}$ & 0 & 1 & 6 & 3 & 4 & 5 & 6 & 4 & 1 & 2 \\
\hline $\mathbf{0}$ & $\mathbf{6}$ & $\mathbf{0 , 1 , 2 , 3 , 4 , 5 , 6}$ & 0 & & 6 & 3 & 4 & 6 & 0 & 5 & 2 & 3 \\
\hline $\mathbf{1}$ & $\mathbf{1}$ & $\mathbf{0 , 1 , 2 , 4 , 5}$ & 1 & 2 & 0 & 4 & 5 & 1 & 2 & 0 & 4 & 5 \\
\hline $\mathbf{1}$ & $\mathbf{2}$ & $\mathbf{0 , 1 , 2 , 3 , 4 , 5 , 6}$ & 1 & 2 & 0 & 4 & 5 & 2 & 3 & 1 & 5 & 6 \\
\hline $\mathbf{1}$ & $\mathbf{3}$ & $\mathbf{0 , 1 , 2 , 3 , 4 , 5 , 6}$ & 1 & 2 & 0 & 4 & 5 & 3 & 4 & 2 & 6 & 0 \\
\hline $\mathbf{1}$ & $\mathbf{4}$ & $\mathbf{0 , 1 , 2 , 3 , 4 , 5}$ & 1 & 2 & 0 & 4 & 5 & 4 & 5 & 3 & 0 & 1 \\
\hline $\mathbf{1}$ & $\mathbf{5}$ & $\mathbf{0 , 1 , 2 , 4 , 5 , 6}$ & 1 & 2 & 0 & 4 & 5 & 5 & 6 & 4 & 1 & 2 \\
\hline $\mathbf{1}$ & $\mathbf{6}$ & $\mathbf{0 , 1 , 2 , 3 , 4 , 5 , 6}$ & 1 & 2 & 0 & 4 & 5 & 6 & 0 & 5 & 2 & 3 \\
\hline $\mathbf{2}$ & $\mathbf{2}$ & $\mathbf{1 , 2 , 3 , 5 , 6}$ & 2 & 3 & 1 & 5 & 6 & 2 & 3 & 1 & 5 & 6 \\
\hline $\mathbf{2}$ & $\mathbf{3}$ & $\mathbf{0 , 1 , 2 , 3 , 4 , 5 , 6}$ & 2 & 3 & 1 & 5 & 6 & 3 & 4 & 2 & 6 & 0 \\
\hline $\mathbf{2}$ & $\mathbf{4}$ & $\mathbf{0 , 1 , 2 , 3 , 4 , 5 , 6}$ & 2 & 3 & 1 & 5 & 6 & 4 & 5 & 3 & 0 & 1 \\
\hline $\mathbf{2}$ & $\mathbf{5}$ & $\mathbf{1 , 2 , 3 , 4 , 5 , 6}$ & 2 & 3 & 1 & 5 & 6 & 5 & 6 & 4 & 1 & 2 \\
\hline $\mathbf{2}$ & $\mathbf{6}$ & $\mathbf{0 , 1 , 2 , 3 , 5 , 6}$ & 2 & 3 & 1 & 5 & 6 & 6 & 0 & 5 & 2 & 3 \\
\hline $\mathbf{3}$ & $\mathbf{3}$ & $\mathbf{0 , 2 , 3 , 4 , 6}$ & 3 & 4 & 2 & 6 & 0 & 3 & 4 & 2 & 6 & 0 \\
\hline $\mathbf{3}$ & $\mathbf{4}$ & $\mathbf{0 , 1 , 2 , 3 , 4 , 5 , 6}$ & 3 & 4 & 2 & 6 & 0 & 4 & 5 & 3 & 0 & 1 \\
\hline $\mathbf{3}$ & $\mathbf{5}$ & $\mathbf{0 , 1 , 2 , 3 , 4 , 5 , 6}$ & 3 & 4 & 2 & 6 & 0 & 5 & 6 & 4 & 1 & 2 \\
\hline $\mathbf{3}$ & $\mathbf{6}$ & $\mathbf{0 , 2 , 3 , 4 , 5 , 6}$ & 3 & 4 & 2 & 6 & 0 & 6 & 0 & 5 & 2 & 3 \\
\hline $\mathbf{4}$ & $\mathbf{4}$ & $\mathbf{0 , 1 , 3 , 4 , 5}$ & 4 & 5 & 3 & 0 & 1 & 4 & 5 & 3 & 0 & 1 \\
\hline $\mathbf{4}$ & $\mathbf{5}$ & $\mathbf{0 , 1 , 2 , 3 , 4 , 5 , 6}$ & 4 & 5 & 3 & 0 & 1 & 5 & 6 & 4 & 1 & 2 \\
\hline $\mathbf{4}$ & $\mathbf{6}$ & $\mathbf{0 , 1 , 2 , 3 , 4 , 5 , 6}$ & 4 & 5 & 3 & 0 & 1 & 6 & 0 & 5 & 2 & 3 \\
\hline $\mathbf{5}$ & $\mathbf{5}$ & $\mathbf{1 , 2 , 4 , 5 , 6}$ & 5 & 6 & 4 & 1 & 2 & 5 & 6 & 4 & 1 & 2 \\
\hline $\mathbf{5}$ & $\mathbf{6}$ & $\mathbf{0 , 1 , 2 , 3 , 4 , 5 , 6}$ & 5 & 4 & 6 & 2 & 1 & 6 & 5 & 0 & 2 & 3 \\
\hline
\end{tabular}




\begin{tabular}{l|l}
6 & 5 \\
\hline
\end{tabular}

0

3

2

6

5

\begin{tabular}{l|l}
0 & 3 \\
\hline
\end{tabular}

2

Based on PDS $(0, \pm 1, \pm 3)$ For EX-OR logical operation

\begin{tabular}{|c|c|c|c|c|c|c|c|c|c|c|c|c|}
\hline $\bar{A}$ & $\mathbf{B}$ & connected nodes & $\mathbf{A}+\mathbf{0}$ & $A+1$ & A-1 & $\mathbf{A + 3}$ & A-3 & $\mathbf{B}+\mathbf{0}$ & $\mathrm{B}+1$ & B-1 & $\mathbf{B}+\mathbf{3}$ & B-3 \\
\hline $\mathbf{0}$ & $\mathbf{0}$ & Not connected & $*$ & $*$ & $*$ & $*$ & $*$ & $*$ & $*$ & $*$ & $*$ & $*$ \\
\hline $\mathbf{0}$ & 1 & $2,3,5,6$ & $*$ & $*$ & 6 & 3 & $*$ & $*$ & 2 & $*$ & $*$ & 5 \\
\hline 0 & 2 & $0,2,4,5$ & 0 & * & $*$ & * & 4 & 2 & * & $*$ & 5 & * \\
\hline $\mathbf{0}$ & 3 & 1,2 & $*$ & 1 & $*$ & $*$ & $*$ & $*$ & $*$ & 2 & $*$ & $*$ \\
\hline $\mathbf{0}$ & 4 & 5,6 & $*$ & $*$ & 6 & $*$ & $*$ & $*$ & 5 & $*$ & $*$ & $*$ \\
\hline $\mathbf{0}$ & 5 & $0,2,3,5$ & 0 & $*$ & $*$ & 3 & $*$ & 5 & $*$ & $*$ & $*$ & 2 \\
\hline $\mathbf{0}$ & 6 & $1,2,4,5$ & $*$ & 1 & $*$ & $*$ & 4 & $*$ & $*$ & 5 & 2 & $*$ \\
\hline 1 & 1 & Not connected & $*$ & $*$ & $*$ & $*$ & $*$ & $*$ & $*$ & $*$ & $*$ & $*$ \\
\hline 1 & 2 & $0, \mathbf{3 , 4 , 6}$ & $*$ & $*$ & 0 & 4 & $*$ & $*$ & 3 & $*$ & $*$ & 6 \\
\hline 1 & 3 & $1,3,5,6$ & 1 & $*$ & $*$ & $*$ & 5 & 3 & $*$ & $*$ & 6 & $*$ \\
\hline 1 & 4 & 2,3 & $*$ & 2 & $*$ & $*$ & $*$ & $*$ & $*$ & 3 & $*$ & $*$ \\
\hline 1 & 5 & 0,6 & $*$ & $*$ & 0 & $*$ & $*$ & $*$ & 6 & $*$ & $*$ & $*$ \\
\hline 1 & 6 & $1,3,4,6$ & 1 & $*$ & $*$ & 4 & $*$ & 6 & $*$ & $*$ & $*$ & 3 \\
\hline 2 & 2 & Not connected & $*$ & $*$ & $*$ & $*$ & $*$ & * & $*$ & $*$ & $*$ & $*$ \\
\hline 2 & 3 & $0,1,4,5$ & $*$ & $*$ & 1 & 5 & $*$ & $*$ & 4 & $*$ & $*$ & 0 \\
\hline 2 & 4 & $0,2,4,6$ & 2 & $*$ & $*$ & $*$ & 6 & 4 & $*$ & $*$ & 0 & $*$ \\
\hline 2 & 5 & 3,4 & $*$ & 3 & $*$ & $*$ & $*$ & $*$ & $*$ & 4 & $*$ & $*$ \\
\hline 2 & 6 & $\mathbf{0 , 1}$ & $*$ & $*$ & 1 & $*$ & $*$ & $*$ & 0 & $*$ & $*$ & $*$ \\
\hline 3 & 3 & Not connected & $*$ & $*$ & $*$ & $*$ & $*$ & $*$ & $*$ & $*$ & $*$ & $*$ \\
\hline 3 & 4 & $1,2,5,6$ & $*$ & $*$ & 2 & 6 & $*$ & $*$ & 5 & $*$ & $*$ & 1 \\
\hline 3 & 5 & $0,1,4,5$ & $*$ & 4 & $*$ & $*$ & 0 & 5 & $*$ & 4 & 1 & $*$ \\
\hline 3 & 6 & 4,5 & $*$ & 4 & $*$ & $*$ & $*$ & $*$ & $*$ & 5 & $*$ & $*$ \\
\hline 4 & 4 & Not connected & $*$ & $*$ & $*$ & $*$ & $*$ & $*$ & $*$ & $*$ & $*$ & $*$ \\
\hline 4 & 5 & $0,2,3,6$ & $*$ & $*$ & 3 & 0 & $*$ & $*$ & 6 & $*$ & $*$ & 2 \\
\hline 4 & 6 & $1,2,4,6$ & 4 & $*$ & $*$ & 1 & 6 & 6 & $*$ & $*$ & 2 & $*$ \\
\hline 5 & 5 & Not connected & $*$ & $*$ & $*$ & $*$ & $*$ & $*$ & $*$ & $*$ & $*$ & $*$ \\
\hline 5 & 6 & $\mathbf{0 , 1 , 3 , 4}$ & $*$ & 4 & $*$ & $*$ & 1 & $*$ & $*$ & 0 & $*$ & 3 \\
\hline 6 & 6 & Not connected & $*$ & $*$ & $*$ & $*$ & $*$ & $*$ & $*$ & $*$ & $*$ & $*$ \\
\hline
\end{tabular}

Based on PDS $(0, \pm 1, \pm 3)$ For Bi-implication logical operation

\begin{tabular}{|l|l|c|c|c|c|c|c|c|c|c|c|c|}
\hline $\mathbf{A}$ & $\mathbf{B}$ & $\mathbf{c o n n e c t e d ~ n o d e s ~}$ & $\mathbf{A + 0}$ & $\mathbf{A + 1}$ & $\mathbf{A - 1}$ & $\mathbf{A + 3}$ & $\mathbf{A - 3}$ & $\mathbf{B + 0}$ & $\mathbf{B + 1}$ & $\mathbf{B}-\mathbf{1}$ & $\mathbf{B + 3}$ & $\mathbf{B}-\mathbf{3}$ \\
\hline $\mathbf{0}$ & $\mathbf{0}$ & $\mathbf{0 , 1 , 2 , 3 , 4 , 5 , 6}$ & 0 & 1 & 6 & 3 & 4 & 0 & 1 & 6 & 3 & 4 \\
\hline $\mathbf{0}$ & $\mathbf{1}$ & $\mathbf{0 , 1 , 4}$ & 0 & 1 & $*$ & $*$ & 4 & 1 & $*$ & 0 & 4 & $*$ \\
\hline $\mathbf{0}$ & $\mathbf{2}$ & $\mathbf{1 , 3 , 6}$ & $*$ & 1 & 6 & 3 & $*$ & 2 & 3 & 1 & 5 & 6 \\
\hline $\mathbf{0}$ & $\mathbf{3}$ & $\mathbf{0 , 3 , 4 , 5 , 6}$ & 0 & $*$ & 6 & 3 & 4 & 3 & 4 & $*$ & 6 & 0 \\
\hline $\mathbf{0}$ & $\mathbf{4}$ & $\mathbf{0 , 1 , 2 , 3 , 4}$ & 0 & 1 & $*$ & 3 & 4 & 4 & 5 & 3 & 0 & 1 \\
\hline $\mathbf{0}$ & $\mathbf{5}$ & $\mathbf{1 , 4 , 6}$ & $*$ & 1 & 6 & $*$ & 4 & 5 & 6 & 4 & 1 & 2 \\
\hline $\mathbf{0}$ & $\mathbf{6}$ & $\mathbf{0 , 3 , 6}$ & 0 & $*$ & 6 & 3 & $*$ & 6 & 0 & 5 & 2 & 3 \\
\hline $\mathbf{1}$ & $\mathbf{1}$ & $\mathbf{0 , 1 , 2 , 3 , 4 , 5 , 6}$ & 0 & 1 & 6 & 3 & 4 & 0 & 1 & 6 & 3 & 4 \\
\hline $\mathbf{1}$ & $\mathbf{2}$ & $\mathbf{1 , 2 , 5}$ & 1 & 2 & $*$ & $*$ & 5 & 2 & $*$ & 1 & 5 & $*$ \\
\hline $\mathbf{1}$ & $\mathbf{3}$ & $\mathbf{0 , 2 , 4}$ & $*$ & 2 & 0 & 4 & $*$ & $*$ & 4 & 2 & $*$ & 0 \\
\hline
\end{tabular}




\begin{tabular}{|c|c|c|c|c|c|c|c|c|c|c|c|c|}
\hline 1 & 4 & $0, \mathbf{1}, \mathbf{4 , 5 , 6}$ & 1 & $*$ & 0 & 4 & 5 & 4 & 5 & $*$ & 0 & 1 \\
\hline 1 & 5 & $1,2,4,5,6$ & 1 & 2 & $*$ & 4 & 5 & 5 & 6 & 4 & 1 & 2 \\
\hline 1 & 6 & $0,2,5$ & $*$ & 2 & 0 & * & 5 & 6 & 0 & 5 & 2 & 3 \\
\hline 2 & 2 & $0,1,2,3,4,5,6$ & 0 & 1 & 6 & 3 & 4 & 0 & 1 & 6 & 3 & 4 \\
\hline 2 & 3 & $2,3,6$ & 2 & 3 & $*$ & $*$ & 6 & 3 & 4 & 2 & 6 & 0 \\
\hline 2 & 4 & $1,3,5$ & $*$ & 3 & 1 & 5 & * & $*$ & 5 & 3 & 0 & 1 \\
\hline 2 & 5 & $0,1,2,5,6$ & 2 & $*$ & 1 & 5 & 6 & 5 & 6 & $*$ & 1 & 2 \\
\hline 2 & 6 & $2,3,4,5,6$ & 2 & 3 & $*$ & 5 & 6 & 6 & $*$ & 5 & 3 & 2 \\
\hline 3 & 3 & $0,1,2,3,4,5,6$ & 0 & 1 & 6 & 3 & 4 & 0 & 1 & 6 & 3 & 4 \\
\hline 3 & 4 & $0,3,4$ & 3 & 4 & $*$ & $*$ & 0 & 4 & $*$ & 3 & $*$ & 0 \\
\hline 3 & 5 & $2,4,6$ & $*$ & 4 & 2 & 6 & $*$ & $*$ & 6 & 4 & 2 & $*$ \\
\hline 3 & 6 & $0,1,2,3,6$ & 3 & $*$ & 2 & 6 & 0 & 6 & 0 & $*$ & 3 & 2 \\
\hline 4 & 4 & $0,1,2,3,4,5,6$ & 0 & 1 & 6 & 3 & 4 & 0 & 1 & 6 & 3 & 4 \\
\hline 4 & 5 & $1,4,5$ & 4 & 5 & $*$ & $*$ & 1 & 5 & $*$ & 4 & $*$ & 1 \\
\hline 4 & 6 & $0,3,5$ & $*$ & 5 & 3 & 0 & $*$ & $*$ & 0 & 5 & 3 & $*$ \\
\hline 5 & 5 & $0,1,2,3,4,5,6$ & 0 & 1 & 6 & 3 & 4 & 0 & 1 & 6 & 3 & 4 \\
\hline 5 & 6 & $2,4,6$ & $*$ & 6 & 4 & $*$ & 2 & 6 & $*$ & $*$ & $*$ & 2 \\
\hline 6 & 6 & $0,1,2,3,4,5,6$ & 0 & 1 & 6 & 3 & 4 & 0 & 1 & 6 & 3 & 4 \\
\hline
\end{tabular}

\section{ALGORITHMIC DEVELOPMENT}

\subsection{The Algorithm setup for AND logical operation for PDN $\delta=2$}

Step1- set node $\mathrm{i}$ and node $\mathrm{j}$

Step 2- for $\mathrm{i}=0$ to $\mathrm{n}$

For $\mathrm{j}=0$ to $\mathrm{n}$

Val $=$ node $\mathrm{i} \wedge$ node $\mathrm{j}$ (bitwise) $\mathrm{j}++$

Print val

$$
\mathrm{I}++
$$

Step 3- stop

The output of the algorithm will generate the following pattern 5,33,44,33. Algorithm for the other logical operation can be given similar way by replacing logical symbol.

\section{CONCLUSION}

We are using logical operators between vectors of connectivity matrix to study connectivity \& complexity of network. We found that binary relation between nodes is well defined with network flow. The principle of Boolean Algebra may holds in interconnection network. The following are the patterns of the bit of the vector of a interconnection network.

1. AND operations of the Vectors of a PDN-5,33,44,33

2. EX-OR operation of the Vectors of PDN-5,77,66,77

3. OR operations of the Vectors of a PDN-0,44,22,44

4. Implication operation of the Vectors of a PDN$7,55,66,55$

5. Bi-Implication operations of the Vectors of a PDN $7,33,55,33$

\section{REFERENCES}

[1] C. Wu and T. Feng. Tutorial, interconnection networks for parallel and distributed processing. Tutorial Texts Series. IEEE Computer Society Press, 1984.

[2] www.interconnection of networks, elements of parallel computing and architecture [Last seen 22-11-2018]

[3] Ms J.Nandagaoli and Dr. J.W. Bakal, "Study of Perfect Difference Network", International journal of Computer Science", Vol 3, Issue 6 July 2014.

[4] Behrooz Parhami, Mikhail Rakov "Application of Perfect Difference Sets to the Design of Efficient and Robust Interconnection Networks".

[5] S.Tiwari and R.K.Katare, "A Study of fabric of Architecture using Structural Pattern and Relation", "International Journal of Latest Technology in Engineering and Management and Applied Science", Vol 4, Issue 09,Sep 2015.

[6] S.Tiwari, R.K.Katare,V. Sharma and C.M.Tiwari, "Study of Geometrical Structure of Perfect Difference Network", “ International Journal of Advanced Research in Computer and Communication Engineering", Vol5,Issue3,March 2016.

[7] Ms J.Nandagaoli and Dr. J.W. Bakal, "Study of Perfect Difference Network", International journal of Computer Science", Vol 3, Issue 6 July 2014.

[8] J. Beiriger, W. Johnson, H. Bivens et al., "Constructing the ASCI Grid," In: 9th IEEE Symposium on High Performance Distributed Computing, IEEE Press, New York, 2000, pp. 193 - 200.

[9] Agarwal, A. and Agarwal, A. (2011). The Security Risks Associated with Cloud Computing. International Journal of Computer Applications in Engineering Sciences, 1 (Special Issue on CNS), 257-259.

[10] Katare R K and Chaudhary N S, "Study of topological property of interconnection networks and its mapping to Sparse Matrix model” International journal, 2009. 
Rakesh Kumar Katare et al, International Journal of Advanced Research in Computer Science, 10 (6), Nov-Dec 2019,29-39

[11] Singer J. "A theorm in Finite Projective Geometry and Some Apllications to Number Theory" Thrans. American Mathematical Society,Vol.43,pp.377-385,1938.

[12] Katare,R.K.,Chaudari,N.S., Mugal,S.A.,Verma,S.K., Imran, S. Raina,R.R. "Study of link Utilization of Perfect
Difference Network and Hypercube "Conference on"FECS",the world congress in Computer Science,Computer Engineering and Applied Computing,Las Vegas,Nevada,USA,July -25,2013 